\title{
Etudes sur les caractères culturaux et la morphologie de Geotrichum candidum Link
}

\author{
par \\ Micheline GUEGUEN* et J. JACQUET*
}

\section{Ré s u m é}

L'étude des caractères culturaux de Geotrichum candidum Link sur différents milieux de cultures solides, a permis de différencier 3 types morphologiques, spécialement sur milieu à l'extrait de malt.

Ils se distinguent également par des différences physiologiques et biochimiques.

Type 1: souches de couleur crème, à l'aspect levuriforme, à température optimale située entre 22 et $25^{\circ} \mathrm{C}$, à croissance plus réduite à $30^{\circ} \mathrm{C}$, à production abondante d'arthrospores, donnant peu de mycelium ; plutôt acidifiantes, à activité protéolytique faible.

Type 2 : intermédiaire.

Type 3 : souches bien blanches, plus ou moins feutrantes, à température optimale plus élevée $\left(25-30^{\circ} \mathrm{C}\right)$, à croissance plus faible à $22^{\circ} \mathrm{C}$, sporulant peu, produisant en milieux liquides des myceliums plus importants que les souches du type 1 ; plutôt alcalinisantes, à activité protéolytique plus marquée.

Ces différences laissent place à une possibilité de sélection pour la fromagerie.

Mots clés

Geotrichum candidum - Morphologie - Caractères culturaux.

Titre abrégé

Caractères culturaux, morphologie de Geotrichum candidum.

\footnotetext{
* Laboratoire de microbiologie et de physico-chimie alimentaires, U.E.R. des Sciences de l'Alimentation et de la Nutrition, Université de Caen - 14032 Caen cedex (France).
} 


\section{S u m m a r y}

\section{STUDIES ABOUT THE CULTURAL CHARACTERISTICS AND THE MORPHOLOGY OF GEOTRICHUM CANDIDUM LINK}

The study about the cultural characteristics of Geotrichum candidum Link in different solid mediums of culture has enabled to differentiate 3 morphologic models, specially in malt extract medium.

They are differentiated too by physiologic and biochemical differences.

Model 1 : Cream-coloured strains, yeast like, with an optimum temperature between 22 and $25^{\circ} \mathrm{C}$, with a lower growth at $30^{\circ} \mathrm{C}$, with a copious production of arthrospores, giving few mycelium; rather acidifying, with a low proteolytic activity.

Model 2 : Intermediary.

Model 3: Well white strains, more or less felting, with a higher optimum temperature $\left(25-30^{\circ} \mathrm{C}\right)$, with a lower growth at $22^{\circ} \mathrm{C}$, little sporulating, producing more important mycelium than the model 1 strains do in fluid medium; rather alkalinizating, with a stronger proteolytic activity.

These differences give a possibility of selection for cheese-dairies. Key words

Geotrichum candidum - Morphology - Cultural characteristics.

Condensed title

Cultural characteristics, Geotrichum candidum morphology.

\section{INTRODUCTION}

Le champignon Geotrichum candidum Link est un des plus répandus dans les milieux acidifiés : produits laitiers, évidemment et ceci avec une très grande fréquence, mais aussi, plaies, muqueuses, sécrétions. Cet hyphomycète appelé naguère Oïdium lactis Fresenius ou Oospora lactis (Fres.) Saccardo (Carmichael, 1957) a comme forme ascosporée le Dipodascus geotrichum Butler et Petersen = Galactomyces geotrichum (Butl. et Pet.) Redhead et Malloch (Butler et Petersen, 1970, 1972 ; Redhead et Malloch, 1977; Onions Allsopp et Eggins, 1981 ; von Arx, 1977). Plusieurs auteurs (Saëz, 1957, Vieu et Segretain, 1959, Morenz, 1963 et 1964, Gueho et Buissière, 1975) considèrent les confusions entre diverses espèces de Geotrichum et même avec des espèces de genres voisins comme très faciles, et notamment les Trichosporon, Endomyces, Protendomycopsis. Mais, c'est $G$. candidum qui est de beaucoup, le plus banal. 
Les travaux qui sont consacrés à sa morphologie sont de plus en plus nombreux maintenant. Les publications de Steele et Fraser en 1973, Trinci en 1974, Fiddy et Trinci en 1976, Prosser et Trinci en 1979, Robinson et Smith en 1979 et 1980, Smith et Robinson en 1980, portent sur la vitesse de croissance des hyphes, leurs ramifications, leur ultrastructure. Ceux de Cole et Kendrick en 1969, Steele en 1973, Steele et Fraser en 1973, Duran, Uruburu et Villanueva également en 1973, Trinci et Collinge en 1974, Cole en 1975, Allermann et al. en 1978, Kier et al, en 1980 mettent l'accent sur la morphologie, la structure, la formation et la germination des arthrospores.

Mais ces auteurs n'ont eu généralement recours qu'à une seule souche*. Ayant pu rassembler près de 150 échantillons provenant surtout de produits laitiers, des aspects morphologiques inédits sont apparus, en même temps que nous étions frappés par la variabilité des formes. Il nous a donc semblé utile de publier ces résultats qui peuvent revêtir une importance pratique non négligeable.

\section{MATERIEL ET METHODES UTILISES}

\section{Souches}

Précisons, tout d'abord, qu'il s'agit en réalité d' "isolats », les thalles comparés n'étant pas issus d'une culture monosporée. Le mot "souche " sera donc à prendre dans son sens large.

Pour déterminer l'aspect des cultures, nous avons disposé de 96 souches de $G$. candidum dont 89 retirées par nous de fromages divers. Quatre souches ont été sorties d'une collection, 3 de celle du Museum d'Histoire Naturelle à Paris (M.H.N.) : ce sont les numéros 577, 590, 2188; une provenait du laboratoire de Botanique de l'Université de Caen : elle est indiquée sous le code VS 56. Nous $\mathrm{y}$ avons adjoint les souches de trois levains industriels commercialisés, qui paraissent être des mélanges (ce sont les Lactolabo no 2 et 8 et le levain Labo-standa).

Pour étudier la vitesse de développement, nous avons mis en jeu 65 souches dont 9 de collection, les 4 précitées et les $n^{\text {os }} 11012$ $11623,13324,18033$ et 24062 du Centraalbureau voor Schimmelcultures de Baarn (C.B.S.).

Parmi ces 65 souches, 20 seront testées pour déterminer la température optimale de croissance et l'influence de basses températures.

Bien que trouvé pour la plupart de ses composants sur des produits laitiers, nous pensons que notre échantillonnage est bien représentatif de l'espèce elle-même et que l'origine n'a modifié en

* Le travail de Carmichael, qui a porté sur 52 souches fait exception, mais son intérêt est surtout d'ordre bibliographique. 
rien les caractéristiques des Geotrichum. Les souches de collection intégrées aux nôtres serviront d'ailleurs de témoins.

L'identification de nos champignons a été effectuée selon les données de Carmichael, et la Clé de Morenz (1964) et contrôlée par le Centraalbureau voor Schimmelcultures de Baarn (Pays-Bas), à qui nous avons adressé les souches $\mathrm{n}^{\circ}$ G 36 et G 116 .

Pour la conservation, nous avons utilisé :

- soit le repiquage en gélose au malt inclinée, tous les 6 à 8 mois, avec mise au frigorifique à $+4^{\circ} \mathrm{C}$ le reste du temps;

- soit la lyophilisation ;

- soit l'ensemencement en tubes renfermant du sable de Fontainebleau stérile.

\section{Milieux de culture et ensemencements}

Pour que les résultats de notre travail théorique soient applicables à la fromagerie, nous n'avons utilisé que des milieux de culture solides, gélosés à $1,8 \%$.

a) Cultures traditionnelles

- milieu nutritif à l'extrait de malt Difco à $30 \mathrm{~g} / 1$;

- milieu de Sabouraud;

- milieu de Czapek-Dox ;

- milieu de Czapek-trypticase*;

- milieu au lactosérum de fromagerie de camembert digéré à la papaïne de titre $80(1 \mathrm{~g} / \mathrm{l})$ pendant $3 \mathrm{~h}$ à $70^{\circ} \mathrm{C}$;

- milieu au lait.

Le pH est réglé, par action de l'acide lactique et de l'ammoniaque de façon à obtenir, au moment de l'emploi, une valeur de 5 ,5. Les milieux sont stérilisés à $115^{\circ} \mathrm{C}$ pendant $20 \mathrm{~min}$.

Pour l'étude des caractères culturaux, les milieux ont été répartis en tubes inclinés et ensemencés en nappe à la pipette Pasteur (3 gouttes) à partir de suspensions d'arthrospores titrant 2 à $4.10^{6}$ spores au $\mathrm{ml}$. Les cultures sont ensuite maintenues une semaine à la température ambiante.

Pour l'appréciation des vitesses de croissance, les milieux ont été coulés en boîtes de Pétri de $90 \mathrm{~mm}$ de diamètre et ensemencés par dépôt, en leur centre, d'un disque d'eau gélosée de $10 \mathrm{~mm}$ de diamètre contenant 3 à $6.10^{3}$ spores. Le diamètre des thalles développés à $24^{\circ} \mathrm{C}$ a été mesuré toutes les $24 \mathrm{~h}$ pendant une semaine.

\footnotetext{
* Trypticase BioMérieux $10 \mathrm{~g}$, glucose $30 \mathrm{~g}, \mathrm{KH}_{2} \mathrm{PO}_{4} 1 \mathrm{~g}, \mathrm{MgSO}_{4} 7 \mathrm{H}_{2} \mathrm{O} 0,5 \mathrm{~g}, \mathrm{KCl}$ $0,5 \mathrm{~g}, \mathrm{FeSO}_{4} 7 \mathrm{H}_{2} \mathrm{O} 0,01 \mathrm{~g}$, eau distillée q.s.p. $1000 \mathrm{ml}$.
} 
En employant la même température d'ensemencement, sur milieu 4,20 souches ont été mises à incuber à 22,25 et $30^{\circ} \mathrm{C}$, pour déterminer la température optimale de croissance; et à $11-12^{\circ} \mathrm{C}$, pour étudier leur comportement à une température voisine de celle des caves d'affinage de certains fromages, comme le St-Nectaire par exemple. La lecture a été effectuée par mesure des diamètres des colonies chaque jour pendant une semaine pour les trois premières températures, et après 11,15 et $24 \mathrm{j}$ pour la température la plus basse.

Pour mesurer les dimensions des arthrospores, un fragment de thalle d'une culture de $4 \mathrm{j}$ à $24^{\circ} \mathrm{C}$ sur milieu à l'extrait de malt est déposé sur une lame de verre. L'empreinte laissée par celui-ci est alors colorée au bleu coton et au lactophénol d'Amann. Vingt-trois souches ont ainsi été examinées, certaines deux fois. Pour chaque souche retenue, le nombre d'éléments mesurés a oscillé entre 50 et 70 .

b) Microcultures sur lames

Pour l'étude de l'influence éventuelle d'une baisse de température de quelques degrés sur la vitesse de germination des arthrospores, la septation et la désarticulation des hyphes, des microcultures ont été réalisées en milieux à l'extrait de malt et Czapek-trypticase, recouvertes d'une lamelle et incubées à $19-20^{\circ} \mathrm{C}$ et à $15-16^{\circ} \mathrm{C}$. Les observations microscopiques ont été faites toutes les demi-heures pendant $5 \mathrm{~h}$, ensuite, toutes les heures pendant $6 \mathrm{~h}$, puis $11 \mathrm{~h}$ plus tard, soit $23 \mathrm{~h}$ après l'ensemencement.

\section{RESULTATS}

\section{A-1. Caractères culturaux}

1. Le milieu a L'EXtRait de MALT permet de regrouper les différentes souches en trois types.

Le premier type présente des thalles ou des plages plats, de couleur crème, qui rappellent un peu des colonies de levures. Bien qu'un spécialiste ne puisse s'y tromper, car l'analogie n'est pas absolue, nous les qualifierons de levuriformes pour la facilité de l'exposé. C'est dans ce groupe que se range la souche CBS 13324 du Centre de Baarn.

Le deuxième type a encore des thalles blanc-crème, mais l'aspect levuriforme est moins marqué. On y aperçoit, d'ailleurs, des hyphes dressés verticalement visibles déjà à l'œil nu, très nets à la loupe binoculaire. La souche MHN 2188 appartient à ce type.

Dans le troisième type, se classent des souches allant d'un feutrage blanc très fin à un feutrage plus ou moins dense. C'est le cas de la souche VS 56 de Caen (presque rase) et 590 (nettement feutrante). 
2. Le Milieu AU LActosérum est remarquable pour les études de microbiologie fromagère ; certains n'hésitent pas à considérer comme constamment transposables directement à la pratique professionnelle les résultats acquis grâce à lui. Il permet des observations similaires à celles du milieu précédent, à la différence près que les modifications d'aspect sont moins accusées.

Les souches du type 1 y forment des thalles légèrement poudreux, blanc-crème à jaunâtres.

Au type 2 correspond un très léger feutrage de couleur blanche.

Le type 3, auquel on passe à partir du type 2 par transitions progressives, montre un feutrage plus ou moins net, plus ou moins serré, mais très blanc, et pouvant prendre aussi un aspect velouté.

3. Le milieu de Sabouraud mis au point pour l'étude des dermatophytes et donc pas forcément adapté à tous les champignons, permet de reconnaître seulement deux types de Geotrichum :

- des souches très rases de couleur jaunâtre, levuriformes ou avec quelques rares hyphes dressés;

- des souches à feutrage blanc et à aspect mycélien plus ou moins net.

Ceci montre bien que le type 2 ne rassemble que des variétés intermédiaires, formant transition entre les morphologies d'aspect 1 et 3 ; sur milieu de Sabouraud, elles se répartissent entre les formes extrêmes.

4. Enfin, LE MILIEU CZAPEK-TRyPticase fait presque toujours perdre aux thalles leur aspect levuriforme, quand il existe. En revanche, les nuances de couleur y sont légèrement plus accusées et la filamentisation plus marquée, si bien que le type 1 se caractérise alors par une présentation d'allure mycélienne, mais seulement légère et parsemée (à l'exception de quelques rares souches qui conservent la morphologie levuriforme que les fromagers qualifient de " graisseuse ") pour arriver à la forme 3 à aspect compact (fig. 1). Certaines souches prennent même une apparence veloutée, voire laineuse, et rappellent ainsi à s'y méprendre les variétés dites « à poil court » de Penicillium camemberti.

\section{A-2. Vitesse de développement de Geotrichum candidum sur milieu solide (en boîtes de Pétri)}

a) Rôle du Milieu de culture

Nous constatons, tout d'abord, que le milieu de Czapek-Dox convient assez mal : la moitié des souches y a une croissance ralentie, l'autre moitié forme un tapis très clairsemé. Les meilleurs milieux sont celui de Czapek-trypticase, ou la gélose au lait. Les nombres ci-dessous montrent les écarts de diamètres en $\mathrm{mm}$ des thalles de 


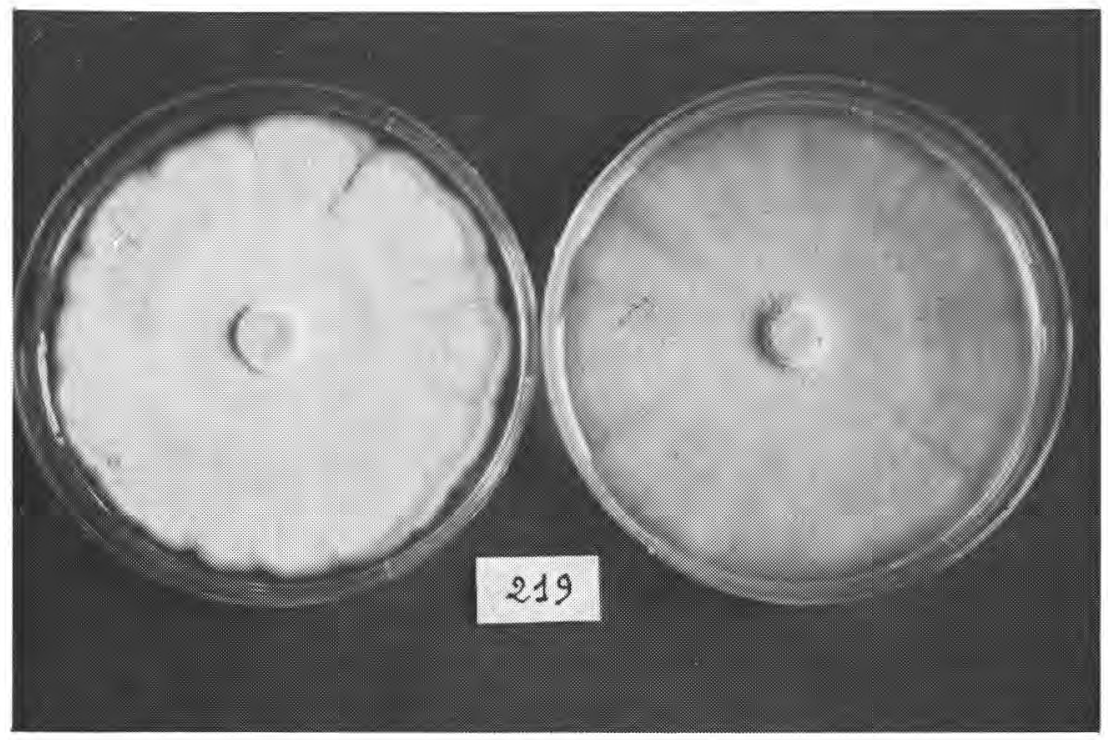

Fig. 1

Aspect des cultures de $G$, candidum envahissant le milieu de Czapek-trypticase à partir d'un ensemencement central en boîtes de Pétri : à gauche souche 819 (type 3 feutrant), à droite souche 5LC (type 1 levuriforme).

Aspect of Geotrichum candidum cultures overspreading the Czapek-trypticase medium from a middle sowing in Petri's boxes: on the left: strain 819 (Model 3, felting), on the right: strain SLC (Model 1, yeast like).

G. candidum après 3 et 7 jours de culture à $24^{\circ} \mathrm{C}$, selon les milieux utilisés.

Milieu de Czapek-trypticase

3 jours

7 jours

L'observation microscopique concommittente nous a montré que le milieu de Czapek-trypticase induisait plus rapidement la désarticulation des hyphes.

Parmi les 65 souches retenues pour cette étude, 11 ont, enfin, été sélectionnées en fonction de leur différence de comportement sur les milieux précédents. Sur gélose au lait, elles sont extrêmement rases, quelquefois très légèrement poudreuses ou filamenteuses. Leur vitesse de développement est pratiquement égale (pour les souches rapides) ou nettement supérieure (pour les souches lentes) à celle obtenue sur Czapek-trypticase.

Bien que la gélose au lait ne modifie pas le classement des souches en fonction de leur vitesse de croissance, elle tend à res- 


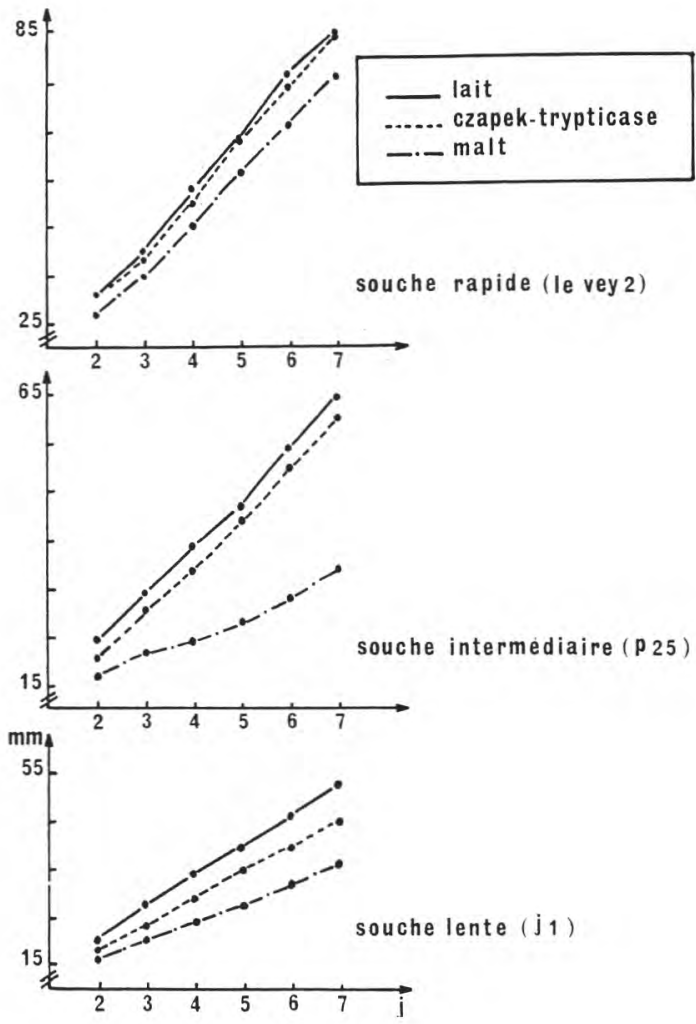

Fig. 2

Vitesse de croissance de diverses souches de G. candidum selon le substrat nutritif.

abs :

temps (en jours),

ord :

diamètre des thalles (en $\mathrm{mm})$.

Growing speed of different Geotrichum candidum strains according to the nutritive substrate. abscissa:

period (in days),

ordinate:

thalles' diameter (in $\mathrm{mm}$ ).

treindre les écarts, comme l'illustrent les graphiques de la figure 2 correspondant à trois souches types.

b) RôLE DE LA TEMPÉRATURE

Nous avons, tout d'abord, déterminé la température optimale de développement de 20 souches appartenant aux 3 types structuraux précités, en utilisant les températures déjà employées par Tubaki (1962) : 22,25 et $30^{\circ} \mathrm{C}$.

Aucune souche n'a son optimum à $22^{\circ} \mathrm{C}$.

En général, les souches de couleur crème et plates du type 1 se développent préférentiellement à $222^{\circ} \mathrm{C}$; une exception : la souche $5 \mathrm{LC}$, dont l'optimum est à $25-30^{\circ} \mathrm{C}$.

Les souches blanches, bien feutrantes (type 3) se développent mieux à $25-30^{\circ} \mathrm{C}$ avec un léger optimum à $30^{\circ} \mathrm{C}$; leur pousse est ralentie à $22^{\circ} \mathrm{C}$.

Les souches de type 2 ont un comportement tantôt comme le type 1, tantôt comme le type 3 . 
Les mêmes souches, placées à $11-12^{\circ} \mathrm{C}$ se développent naturellement beaucoup plus lentement. Les diamètres extrêmes en mm obtenus après 11,15 et $24 \mathrm{j}$ d'incubation sont alors les suivants :
11 jours
25-35
15 jours
$31-46$
24 jours
45-67

Pour obtenir les mêmes tailles, à leur température optimale, les mêmes souches, eûssent demandé respectivement :

$\begin{array}{ll}21 / 2-4 \text { jours } & \text { pour } 25-35 \mathrm{~mm} \\ 3-5 \text { jours } & \text { pour } 31-46 \mathrm{~mm} \\ 5-8 \text { jours } & \text { pour } 45-67 \mathrm{~mm}\end{array}$

Il faut remarquer, enfin, que les souches que nous avons classées primitivement comme rapides à leurs températures normales de développement sont encore celles qui se développent le plus vite à $11-12^{\circ} \mathrm{C}$. Parallèlement, les souches dites lentes accusent encore un retard de développement à température basse.

De surcroît, la température d'incubation ne modifie en rien les caractères culturaux, par conséquent l'appartenance à l'un des trois types que nous avons définis.

Nous avons, aussi, suivi les vitesses relatives de développement de 6 souches de $G$. candidum appartenant aux 3 types, par observations microscopiques de microcultures, (cf. méthode II.2.b), sur milieu à l'extrait de malt. Trois souches, représentatives de chaque type, ont été étudiées, dans les mêmes conditions, sur milieu Czapek-trypticase.

A $19-20^{\circ} \mathrm{C}$, les tubes germinatifs apparaissent sur quelques spores en $4 \mathrm{~h}$ à $4 \mathrm{~h} 30$ après l'ensemencement, et ceci pour toutes les souches. Leur formation est, le plus souvent subapicale, quelquefois cependant équatoriale, certaines spores développant deux tubes germinatifs de longueur inégale. Une seule différence a été constatée d'un milieu à l'autre : le nombre de spores germées est plus important sur le milieu 4.

Une heure après germination, les hyphes mycéliens ont atteint une longueur moyenne de $20 \mu \mathrm{m}$. Mais il en existe certains (environ $10 \%$ ) qui sont déjà beaucoup plus longs (55 à $60 \mu \mathrm{m})$.

8 h à 8 h 30 après l'ensemencement, les hyphes atteignent en moyenne $60 \mu \mathrm{m}$ sur milieu au malt; 70 à $100 \mu \mathrm{m}$ sur Czapek-trypticase et le cloisonnement y commence déjà. Il accuse en général un retard de 1 à $2 \mathrm{~h}$ sur milieu au malt. $23 \mathrm{~h}$ après l'ensemencement, les arthrospores sont formées, atteignant leurs tailles définitives, celles qui occupent une position apicale ou subapicale étant plus courtes que les autres.

Il est à remarquer que tous les hyphes cloisonnés ne se désarticulent pas en arthrospores. Cette désarticulation est en particulier peu fréquente chez les souches du type 3. 
Lorsque l'on fait incuber les lames à $15-16^{\circ} \mathrm{C}$, l'apparition du tube germinatif est légèrement retardée (d'une demi-heure environ).

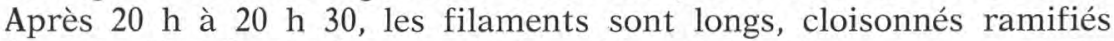
(ce caractère étant plus accentué sur Czapek-trypticase que sur malt). Il n'y a pas encore d'arthrospores. Seule, la souche VS 56 présente un début de désarticulation.

Si l'on replace à ce moment-là, les lames à $19-20^{\circ} \mathrm{C}$, on constate, que seules les souches mises sur milieu de Czapek-trypticase arrivent à former des arthrospores 5 à $6 \mathrm{~h}$ après réchauffement, à l'exception de la souche G 813 qui, elle, en forme toujours, quel que soit le milieu de culture.

Pour que la désarticulation soit constatée sur toutes les souches que nous avons étudiées, il faudra encore attendre plusieurs heures. Mais, cette formation de spores est toujours peu importante chez les souches très feutrantes.

L'observation microscopique montre que ce retard de croissance, induit par l'abaissement de la température et difficilement rattrapable, porte sur la maturation même des hyphes et non sur la phase de germination.
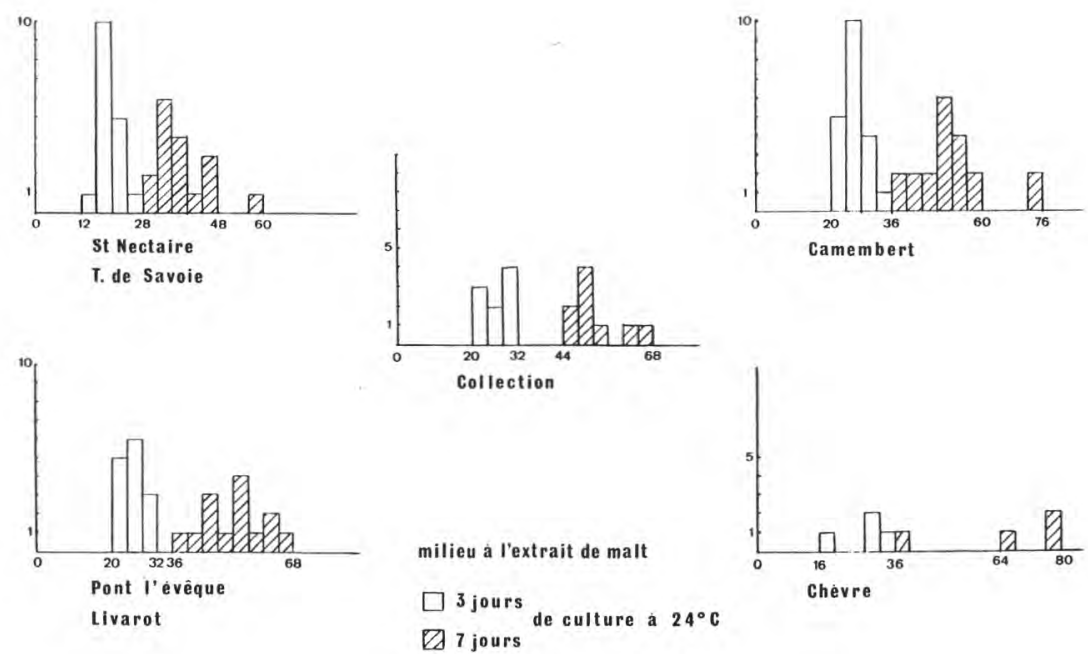

Fig. 3

Fréquence relative des souches à croissance plus ou moins rapide sur milieu à l'extrait de malt selon les types de fromages d'où elles ont été isolées.

abs : diamètre des thalles en $\mathrm{mm}$, ord: nombre de souches.

Relative frequency of strains more or less growing in medium with malt extract according to the cheeses' model from which they are isolated. abscissa: thalles' diameter (in $\mathrm{mm}$ ). ordinate: strains' number. 


\section{A-3. Rapport entre les aspects morphologiques et l'origine des Geotrichum candidum}

Nous avons rassemblé dans un tableau et les histogrammes des figures 3 et 4 l'étude comparative de répartition de nos souches de Geotrichum, en fonction de leur origine, leur aspect morphologique et leur vitesse de croissance.

Le tableau 1 met en évidence la fréquence plus élevée du type 1 dans les fromages à pâte pressée, et une égale répartition des types 1, d'une part, et des types 2-3, d'autre part, dans ceux à pâte molle.

Quant aux vitesses de croissance, elles montrent une homogénéité inattendue dans les variétés rencontrées, sur un même type de fromage, et tout particulièrement, avec les fromages de SaintNectaire et de Tomme de Savoie qui se rapprochent singulièrement l'un de l'autre du point de vue microbiologique. Les variétés isolées à partir de fromages à pâte pressée sont, dans l'ensemble, plus lentes que celles provenant de pâtes molles et les souches de camembert ont un comportement similaire à celles du Pont-l'Evêque.

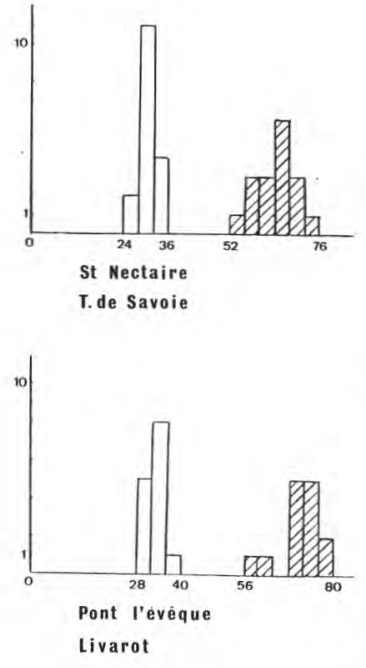

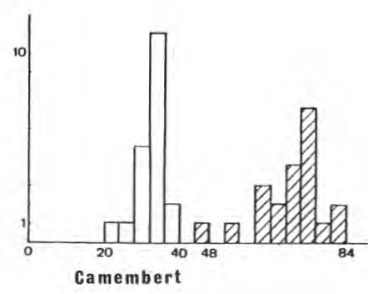

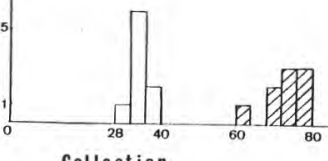

Collection
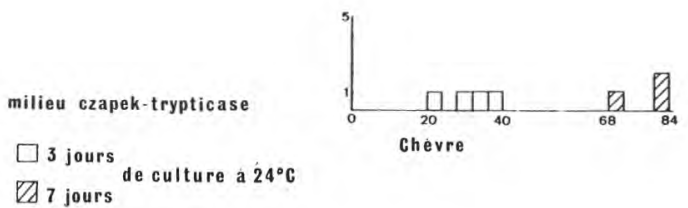

Fig. 4

Fréquence relative des souches à croissance plus ou moins rapide sur milieu de Czapek-trypticase selon leur origine. abs : diamètre des thalles en $\mathrm{mm}$, ord: nombre de souches.

Relative frequency of strains more or less growing in Czapek-trypticase medium according to their origins. abscissa: thalles' diameter (in $\mathrm{mm}$ ). ordinate: strains' number. 


\section{TABLEAU 1 - TABLE 1}

Répartition des types de Geotrichum candidum et de quelques espèces voisines selon leur origine

Distribution of Geotrichum candidum models and of several closely related species according to their origin

\begin{tabular}{|c|c|c|c|c|c|c|}
\hline \multirow[b]{2}{*}{$\begin{array}{c}\text { Origine } \\
\text { des souches }\end{array}$} & \multicolumn{2}{|c|}{ TYPE 1} & \multicolumn{2}{|c|}{ TYPE 2} & \multicolumn{2}{|c|}{ TYPE 3} \\
\hline & $\begin{array}{l}\text { nb. de } \\
\text { souches }\end{array}$ & $\%$ & $\begin{array}{l}\text { nb. de } \\
\text { souches }\end{array}$ & $\%$ & $\begin{array}{l}\text { nb. de } \\
\text { souches }\end{array}$ & $\%$ \\
\hline $\begin{array}{l}\text { FROMAGES } \\
\text { - Camembert } \\
\text { - Pont l'évêque } \\
\text { - Livarot } \\
\text { - Chèvre }\end{array}$ & $\begin{array}{r}26 \\
15 \\
5 \\
2\end{array}$ & & $\begin{array}{l}3 \\
6 \\
0 \\
2\end{array}$ & & $\begin{array}{r}0 \\
25 \\
0 \\
0\end{array}$ & \\
\hline Pâtes molles & 48 & 52 & 11 & 12 & 33 & 36 \\
\hline $\begin{array}{l}\text { - Saint-Nectaire } \\
\text { - Tomme de Savoie }\end{array}$ & $\begin{array}{r}16 \\
2\end{array}$ & & $\begin{array}{l}6 \\
0\end{array}$ & & $\begin{array}{l}4 \\
0\end{array}$ & \\
\hline Pâtes pressées & 18 & 64 & 6 & 22 & 4 & 14 \\
\hline $\begin{array}{l}\text { AUTRES PRODUITS LAITIERS } \\
\text { De gauche à droite : } \\
\text { beurre, crème, yaourt }\end{array}$ & 1 & & 1 & & 1 & \\
\hline $\begin{array}{l}\text { LEVAINS COMMERCIAUX } \\
\text { DE FROMAGERIE }\end{array}$ & 3 & & 1 & & 5 & \\
\hline $\begin{array}{l}\text { CoLLECTION } \\
\text { - Camembert MHN } 577 \\
\text { - Ö̈dium lactis MHN } 2188 \\
\text { souche industrielle lé- } \\
\text { guée par l'INRA } \\
\text { - Tomates CBS } 11623 \\
\text { - Grains d'orge CBS } 24062 \\
\text { - Sol sableux (Burgos, } \\
\text { Espagne) MHN 590 } \\
\text { - Pathogènes présumées: } \\
\text { o I.P. (Paris) CBS } 11012 \\
\text { o Oïdium asteroides } \\
\text { CBS } 13324 \\
\text { o Geotrichum natalense } \\
\text { CBS } 18033 \\
\text { - Vase salée (Sallenelles, } \\
\text { Calvados, France) VS } 56\end{array}$ & 1 & & 1 & & $\begin{array}{l}1 \\
1 \\
1\end{array}$ & \\
\hline Total & 71 & 50 & 21 & 15 & 49 & 35 \\
\hline
\end{tabular}


La dispersion des souches selon leur vitesse de croissance, s'accentue en fonction du temps. Elle est plus prononcée sur milieu à l'extrait de malt que sur Czapek-trypticase.

\section{B. Spores}

Des cultures de $4 \mathrm{j}$ ont été effectuées sur milieu à l'extrait de malt (et non sur Czapek-trypticase) en raison de la généralisation de son emploi, même si d'un laboratoire à l'autre la quantité d'extrait de malt ajouté est variable.

Notons, tout de suite, qu'il y a un lien remarquable entre la morphologie telle que nous l'avons décrite et la facilité de récolte des arthrospores. Après adjonction d'eau physiologique dans les tubes de culture, une simple agitation mécanique, même légère, permet d'obtenir une suspension dense de spores chez les souches levuriformes. Chez les autres et surtout celles du type 3, l'agitation ne peut suffire et il faut avoir recours à un grattage.

Cette différence de comportement ne tient pas à une différence de maturité provenant d'une vitesse de croissance plus ou moins rapide, mais à une proportion dissemblable d'hyphes végétatifs et d'hyphes sporulants que le temps ne modifiera pas sensiblement, ce que l'examen des microcultures nous a démontré.

Nous avons procédé à plus de 1500 mensurations d'éléments unicellulaires, de formes variables, obtenus comme décrit en II.2.a.

Nos résultats montrent les variations de tailles et de formes ci-dessous :

Type 1 : spores de 2 à $5 \mu \mathrm{m} \times 3$ à $18 \mu \mathrm{m}$, moyenne : $3 \times 7,5 \mu \mathrm{m}$, grande variabilité de formes : dale ;

- prédominance d'éléments ovalaires renflés de forme ellipsoï-

- quelques petites formes plus ou moins arrondies (5 à $15 \%$ ) ;

- exceptionnellement, éléments fins, longs, rectangulaires ou de forme cylindrique ( 2 à $8 \%$ ).

Type 2 : spores de 2 à $5 \mu \mathrm{m} \times 3$ à $20 \mu \mathrm{m}$ moyenne : $2,8 \times 8,2 \mu \mathrm{m}:$

- augmentation de la proportion d'éléments fins, rectangulaires (10 à $40 \%$ selon les souches);

- quelques petites formes arrondies.

Type 3 : spores de 2 à $5 \mu \mathrm{m} \times 3,5$ à $25 \mu \mathrm{m}$, moyenne : $2,5 \times 9,1 \mu \mathrm{m}:$

- très peu de formes ovalaires (5 à $20 \%$ );

- grande majorité d'éléments rectangulaires de longueur variable ; 
- rares éléments petits et arrondis.

La figure 5 illustre la différence de proportion des éléments ovalaires et rectangulaires, en fonction de l'appartenance à l'un des 3 types.

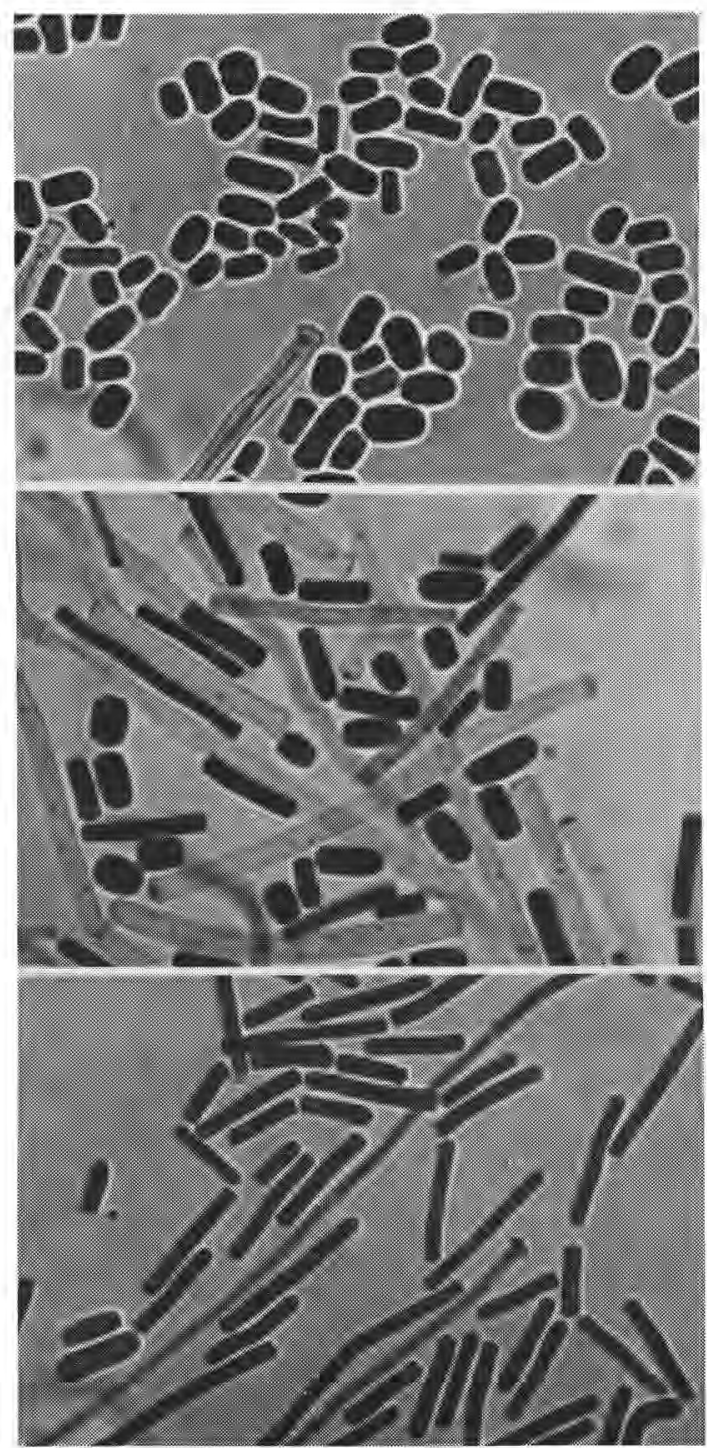

Fig. 5

Aspect au microscope (grossissement 800) de 3 souches appartenant aux différents types :

- en haut, souche G36 (type 1, levuriforme),

- au milieu, souche G 822 (type 2, intermédiaire),

- en bas, souche P 25 (type 3 , feutrant).

Culture de 4 jours à $24^{\circ} \mathrm{C}$, sur milieu à l'extrait de malt.

Aspect in microscope (magnification 800) of 3 strains belonging to the different models:

- at the top, strain $G 36$ (model 1, yeast like),

- at the half-page, strain $G 822$ (model 2, intermediary),

- at the bottom of the page, strain P 25 (model 3 , felting).

4 days' culture in $24^{\circ} \mathrm{C}$ on a maltextract medium. 
Nous parlons ici volontairement d'éléments et non d' " arthrospores », mot qui a une définition bien précise, certains des éléments mensurés n'étant pas des arthrospores au sens strict (voir discussion IV).

Il apparaît une légère augmentation de la longueur moyenne des spores du type 1 à 3 , alors que la largeur diminue. Les spores du premier type sont donc en majorité de forme ellipsoïdale, celles du troisième type surtout de forme cylindrique.

\section{DISCUSSION}

Notre expérience étant établie sur un grand nombre d'échantillons de $G$. candidum, nous amène à formuler des conclusions qui sont forcément différentes de celles de beaucoup d'auteurs précédents.

Tout d'abord, nous notons que le choix des milieux de culture commande jusqu'à un certain point l'aspect morphologique : celui qui exalte le mieux les différences est le milieu à l'extrait de malt ; le milieu qui atténue le plus les variations de forme est celui de Sabouraud. Les meilleures croissances sont, en revanche, obtenues sur Czapek-trypticase.

Il n'y a, cependant, pas de corrélations absolues entre la morphologie, donc l'appartenance à un type, et la vitesse de croissance. $\mathrm{Si}$, parmi les isolats étudiés, aucun souche lente ne nous est apparue très feutrante, en revanche, nous avons trouvé quelques souches rapides qui sont levuriformes.

Notre séparation des représentants de l'espèce $G$. candidum est confortée par ce que nous avons établi de leurs caractères physiologiques et biochimiques où nous trouvons une bonne corrélation, bien que certains auteurs n'hésitent pas à séparer des variétés sur ces seuls critères sans aucune différence morphologique. Nos recherches antérieures (Guéguen et Lenoir, 1975) ont établi, en effet, que les souches du type 1 ont tendance à se développer à la fois à la surface et dans les milieux liquides, à y donner peu de mycélium (mesuré en poids $\mathrm{sec}$ ), à être plutôt acidifiantes et à montrer des activités protéolytiques faibles. Les formes du type 3 ont tendance à se développer surtout à la surface des milieux liquides, à être plutôt alcalinisantes, à avoir une bonne croissance mycélienne et à montrer une nette activité protéolytique.

Il y a, aussi, une différence de température optimale, les souches de type 1 ayant pour celle-ci une valeur plus basse que celles du type 3 ; mais aucun de nos isolats n'a sa croissance maximale à $22^{\circ} \mathrm{C}$, contrairement à ceux de Tubaki (1962). Nous ne pouvons pas confirmer, non plus, l'effet d'accélération de la chaleur sur la crois- 
sance entre 20 et $30^{\circ} \mathrm{C}$, notée par Robinson et Smith, 1979, mais sur une seule souche.

Parmi les auteurs antérieurs qui se sont préoccupés des variariations morphologiques des Geotrichum, Saez (1957) n'indique qu'une seule forme, blanc sale à blanc jaunâtre. Notre type 3 lui est inconnu. Vieu et Segretain (1959) séparent deux aspects parmi les espèces rencontrées dans les prélèvements pathologiques : une $\mathrm{G}_{1}$, la plus fréquente, à colonies blanches poudreuses et relevant de $G$. asterö̈des, l'autres $\mathrm{G}_{2}$, à colonies incolores ou crème qui sont, cette fois, de vrais $G$. candidum. Mais, en quelques mois, $G_{1}$ rejoint l'aspect de $\mathrm{G}_{2}$. Nous n'avons, en ce qui nous concerne, jamais observé une telle transformation, alors que nos souches les plus anciennes sont entretenues au laboratoire depuis 11 ans; les caractères sont stables chez plus de $90 \%$ d'entre elles. Environ $8 \%$ seulement, sont passées, de 1 à 3 fois, après repiquage et toujours d'une façon transitoire, d'un type à un autre sans qu'il n'y ait un sens privilégié. Tubaki (1962) distingue 3 variétés sur des Geotrichum provenant des eaux résiduaires, mais nos 3 types s'apparentent, sans pour autant s'identifier à elles, à ses variétés I et II. Quant à Guého et Buissière (1975) ils fondent leur reconnaissance de 4 biotypes uniquement sur des critères d'ordre enzymatiques et non morphologiques et tous leurs Geotrichum ont une croissance rapide $(24 \mathrm{~h})$ en présence de 6 à $10 \%$ de sel!

La formation des spores, leur morphologie, leur taille, leur germination pourrait appeler une longue discussion que nous ne pouvons qu'amorcer ici.

De nombreux auteurs, tels Carmichael (1957), Saez (1957 et 1969), Butler (1960), Cole et Kendrick (1969), Trinci et Collinge (1974), Cole (1975), Kier et al. (1976 et 1980), Robinson et Smith (1979), Allermann et al. (1978-1980), ont signalé des modifications de forme et de disposition des arthrospores, avec l'âge des cultures, ou la concentration en glucose du milieu.

Les arthrospores, qui se forment par simple désarticulation des hyphes sont, d'abord, cylindriques; puis s'arrondissent à leurs extrêmités, pour se «ballonniser » et devenir plutôt ovalaires.

Pour notre part, nous avons observé trois sortes d'éléments unicellulaires en proportions variables dans les trois types de G. candidum :

$1^{\circ}$ des éléments aux extrémités très arrondies, ou carrément ovalaires, qui correspondent aux formes ellipsoïdales précédemment décrites ; certains, de taille plus importante et très renflés, ressemblant, comme le signalait Saez (1957), à de grosses blastospores.

$2^{\circ}$ des éléments de petite taille, à forme presque ronde, présents chez de nombreuses souches, en quantité réduite, et qui semblent 
correspondre aux endospores décrites par Saez (1969), l'âge des cultures ( $4 \mathrm{j})$, la fréquence d'apparition $(80 \%)$ coïncidant avec nos observations.

Selon les travaux de Cole et Kendrick (1969) et de Cole (1975), seuls les champignons entérothalliques produiraient des endoarthrospores, alors que les holothalliques, auquels appartient G. candidum, forment des arthrospores chez lesquelles toutes les couches de la paroi de la cellule conidiogène sont impliquées dans la formation de la paroi de la spore. Il nous semble qu'il n'y a pas vraiment opposition, nos espèces pouvant produire, à la fois, des arthrospores selon la définition de Cole (1975), et des endospores comme le pense Saez (1957).

$3^{\circ}$ existent enfin des éléments fins, rectangulaires, en proportion majoritaire chez les souches bien feutrantes, qui ne s'arrondissent pas, même chez les cultures les plus âgées :

- Ou bien il s'agit d'arthrospores de forme cylindrique, étroites, et, longues.

- Ou bien ce sont, comme nous le pensons et comme le montre la figure 6 , des éléments d'hyphes végétatifs, relativement courts, et d'où les spores de taille normale se sont séparées à chaque bout.

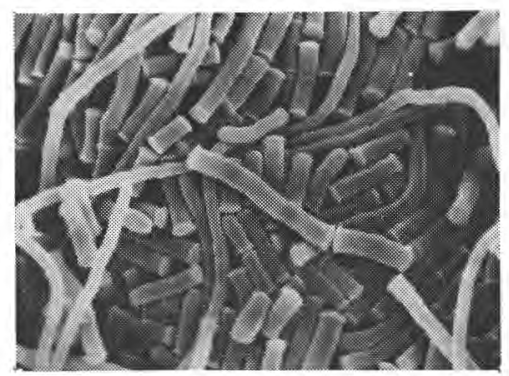

Fig. 6

Aspect au microscope électronique à balayage des arthrospores et des filaments de G. candidum ; souche $7 \mathrm{~s} 9$.

Culture de 3 jours sur milieu à l'extrait de malt à $24^{\circ} \mathrm{C}$ (grossissement $\simeq 1150$ ).

Aspect of Geotrichum candidum arthrospores and filaments in electronic scanning microscope. Strain $7 s 9$.

3 days culture in malt extract medium in $24^{\circ} \mathrm{C}(\mathrm{x} \simeq 1150)$.

Nous n'avons pas eu l'occasion d'observer les « spores levuriformes " de Duran, Uruburu et Villanueva (1973), produites dans certaines conditions de culture plus ou moins défavorables, en particulier à des $\mathrm{pH}$ inférieurs à 3,5 ; ni les modifications induites par les divers taux de glucides; notre préoccupation, malgré l'aspect théorique de ce travail, restant d'obtenir des résultats utilisables dans l'indusrie laitière. Nous n'avons donc pas créé de conditions 
anormales de culture, comme un $\mathrm{pH}$ exagérément bas ou un excès de sucre.

La séparation en 3 types morphologiques dépend d'abord, à notre avis, de la proportion relative d'hyphes sporulants par rapport aux hyphes végétatifs (fig. 7). Interviennent, aussi, l'orientation des

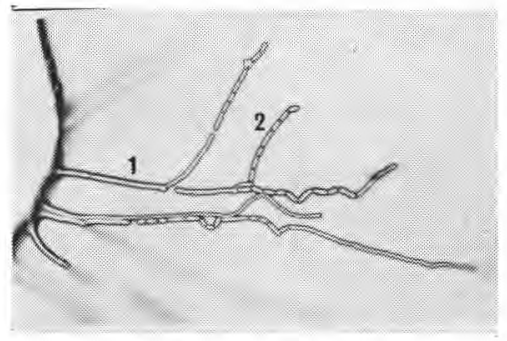

Fig. 7

Microculture de $17 \mathrm{~h}$ à $24^{\circ} \mathrm{C}$ sur milieu à l'extrait de malt, souche $\mathrm{ClF}$.

1. Hyphes végétatifs encore très peu cloisonnés.

2. Hyphes sporulants.

Micro-culture in $24^{\circ} \mathrm{C}$ during 17 hours on malt-extract medium. Strain ClF. 1. Vegetative hyphae yet not very septated.

2. Sporulating hyphae.

hyphes et des chaînes de spores qui peuvent être, soit aériennes, soit couchées à la surface du milieu. Nos observations de boîtes de Pétri, à la loupe binoculaire, $40 \mathrm{~h}$ après l'ensemencement, montrent très nettement ce fait que Carmichael (1957) avait, d'ailleurs, signalé.

En ce qui concerne la germination des arthrospores nos microcultures sur lame ont montré que la vitesse d'apparition du tube germinatif était relativement indépendante du milieu, et peu modifiée par un abaissement de température de 20 à $16^{\circ} \mathrm{C}$. En revanche, le cloisonnement des hyphes (septation) et la désarticulation, sont influencés, à la fois par la température et par le substrat nutritif; le milieu Czapek-trypticase induit ces deux phases plus rapidement que ne le fait la gélose à l'extrait de malt.

Une dernière considération, enfin, mérite d'être soulignée et avec force : quel que soit le milieu, voire même la température, il y a des souches à caractères très nettement individualisés et fixes ce qui laisse place à une possibilité de sélection en fonction de tel ou tel caractère désiré : couleur, vitesse de croissance, aspect des colonies, résistance ou sensibilité thermique relative, capacité de protéolyse, pouvoir feutrant ou apparence levuriforme... En ce qui concerne ce dernier point, nous avons vérifié que l'aspect (soit levuriforme, soit feutrant, soit intermédiaire) restait indépendant $\mathrm{du} \mathrm{pH}$ au moins pour les valeurs com?atibles avec les pratiques fromagères (4 à 7). 


\section{Remerciements}

Nous remercions Mme Sylviane Lemarinier pour la photographie au microscope électronique à balayage.

\section{Bibliographie}

ARX (J. A.) von (1977), - Notes on Dipodascus, Endomyces and Geotrichum with the description of two new species. Antonie van Leeuwenhoek, 43, 333-340.

Allermann (K.), Floto (F.), Olsen (J.), Sortkjaer (O.) and Kier (I.) (1978). Sporulation and the macromolecular composition of the mycelium and arthrospores of Geotrichum candidum. Physiol. Plant., 42, 355-358.

Butler (E. E) (1960). - Pathogenicity and taxonomy of Geotrichum candidum. Phytopath., 50, 665-672.

Butler (E. E.) and Petersen (L. J.) (1970). - Sexual reproduction in Geotrichum candidum. Science, 169, 481-482.

Butler (E. E.) and Petersen (L. J.) (1972), - Endomyces geotrichum a perfect state of Geotrichum candidum. Mycologia, 64, 365-374.

Butler (E. E.), Webster (R. K.) and Eckert (J. W.) (1965). - Taxonomy, pathogenicity and physiological properties of the fungus causing sour rot of Citrus. Phytopath., 55, 1262-1268.

CARmichael (J. W.). (1957). - Geotrichum candidum. Mycologia, 49, 820-830.

Cole (G. T.) (1975). - The thallic mode of conidiogenesis in the Fungi Imperfecti. Can. J. Bot., 53, 2983-3001.

Cole (G. T.) and Kendrick (W. B.) (1969). - Conidium ontogeny in hyphomycestes. The arthrospores of Oidiodendron and Geotrichum, and the endoarthrospores of Sporendonema. Can. J. Bot., 47, 1773-1780.

Duran (A.), Uruburu (F.) and Villanueva (J.) (1973). - Morphogenetic and nutritional studies of Geotrichum lactis cells. Arch. Mikrobiol., 88, 245-256.

FidDY (C.) and Trincr (A. P.) (1976). - Nuclei, septation, branching and growth of Geotrichum candidum. J. Gen. Microbiol., 97, 185-192.

Gueguen (M.) et Lenoir (J.) (1975). - Aptitude de l'espèce Geotrichum candidum à la production d'enzymes protéolytiques. Le Lait, 55, 145-162.

Gueguen (M.) et Lenoir (J.) (1975). - Aptitude de l'espèce Geotrichum candidum à la production d'enzymes protéolytiques. Note complémentaire. Le lait, 55, 621-629.

GuÉHo (E.) et Buissière (J.) (1975). - Méthode d'identification biochimique de champignons filamenteux arthrosporés appartenant au genre Geotrichum Link ex Pers. Ann. Microbiol., 126 A, 483-500.

Kier (I.), Floto (F.), Olsen (J.) and Allermann (K.) (1980). - Macromolecular composition of the wall and protoplasm of mycelia and arthrospores of Geotrichum candidum. Trans. Br. Mycol. Soc., 75, 355-362.

Kier (I.), Allermann (K.), Floto (F.), Olsen (J.) and Sortkjaer (O.) (1976). Changes of exponential growth rates in relation to differentiation of Geotrichum candidum in submerged cultures. Physiol. Plant., 38, 6-12.

Morenz (J.) (1963). - Geotrichum candidum Link. Taxonomic Diagnose und medizinische Bedeutung. Mikol. Schrift., 1, 1-79.

Morenz (J.) (1964). - Taxonomische Untersuchungen zur Gattung Geotrichum. Mikol. Schrift., 2, 33-64.

Onions (A. H.), Allsopp (D.) and EgGins (H. O.) (1981). - Smith's Introduction to industrial Mycology. 1 vol., $7^{\mathrm{e}}$ ed., E. Arnold, Londres. 
PARK (D.) and RoBinson (P. M.) (1970). - Germination studies with Geotrichum candidum. Trans. Br. Mycol. Soc., 54, 83-92.

Prosser (J. I.) and Trinci (A. P.) (1979). - A model for hyphal growth and branching. J. Gen. Microbiol., 111, 153-164.

RedheAd (S. A.) and Malloch (D. W.) (1977), - The endomycetaceae: new concept: new taxa. Cand. J. Bot., 55, 1701-1711.

RoBinson (P. M.) (1980). - Autotropism in germinating arthrospores of Geotrichum candidum. Trans. Br. Mycol. Soc., 75, 151-169.

Robinson (P. M.) and Sмiтh (J. M.) (1979). - Development of cells and hyphae of Geotrichum candidum in chemostat and batch culture. Trans. Br. Mycol. Soc., 72, 39-47.

Robinson (P. M.) and SMITH (J. M.) (1980). - Apical branch formation and cyclic development in Geotrichum candidum. Trans. Br. Mycol. Soc., 75, 233-238.

SAEZ (H.) (1957). - Le Geotrichum candidum Link, caractéristiques morphobiologiques ; fréquence chez l'homme. Bull. Soc. Mycol. France, 73, 343-353.

SAEZ (H.) (1969). - Formation d'endospores chez Geotrichum candidum. Ann. Parasit., 44, 197-204.

Smith (J. M.) and Robinson (P. M.) (1980). - Development of somatic hyphae of Geotrichum candidum. Trans. Br. Mycol. Soc., 74, 159-165.

Steele (S. D.) (1973). - Self-inhibition of arthrospores germination in Geotrichum candidum. Can. J. Microbiol., 19, 943-947.

STEELE (S. D.) and Fraser (T. W.) (1973). - Ultrastructural changes during germination of Geotrichum candidum arthrospores. Can. J. Microbiol., 19, 1031-1034.

SteEle (S. D.) and Fraser (T.W.) (1973). - The ultrastructure of Geotrichum candidum hyphae. Can. J. Microbiol., 19, 1507-1512.

TRINCI (A. P.), (1974). - A study of the kinetics of hyphal extension and branch initiation of fungal mycelia. J. Gen. Microbiol., 81, 225-236.

Trinci (A. P.) and Collinge (A. J.) (1974). - Spore formation in nitrogen and carbon starved cultures of Geotrichum candidum and Mucor racemosus. Trans. Br. Mycol. Soc., 62, 351-358.

TubaKI (K.) (1962). - Studies on a slime-forming fungus in polluted water. Trans. Myc. Soc. Jap., 3, 29-35.

Vieu (M.) et Segretain (G.) (1959). - Contribution à l'étude de Geotrichum et Trichosporum d'origine humaine. Ann. Inst. Pasteur, 96, 421-433. 